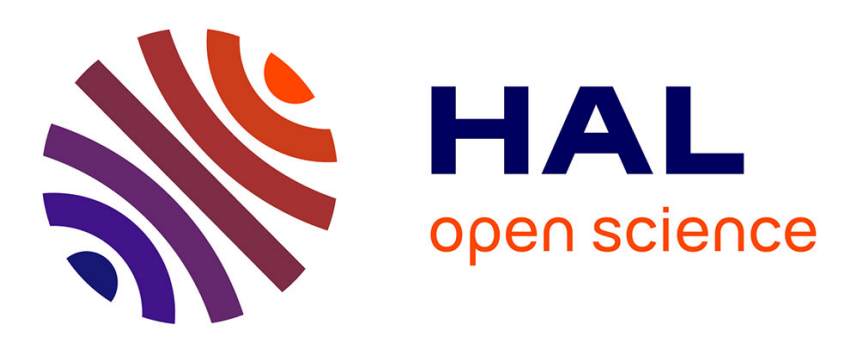

\title{
Sharp acoustic multipolar-resonances in highly monodisperse emulsions
}

Thomas Brunet, Simon Raffy, Benoît Mascaro, Jacques Leng, Régis Wunenburger, Olivier Mondain-Monval, Olivier Poncelet, Christophe Aristégui

\section{- To cite this version:}

Thomas Brunet, Simon Raffy, Benoît Mascaro, Jacques Leng, Régis Wunenburger, et al.. Sharp acoustic multipolar-resonances in highly monodisperse emulsions. Applied Physics Letters, 2012, 101 (1), pp.011913 (1-4). 10.1063/1.4733615 . hal-00731834

\section{HAL Id: hal-00731834 \\ https://hal.science/hal-00731834}

Submitted on 12 Apr 2018

HAL is a multi-disciplinary open access archive for the deposit and dissemination of scientific research documents, whether they are published or not. The documents may come from teaching and research institutions in France or abroad, or from public or private research centers.
L'archive ouverte pluridisciplinaire HAL, est destinée au dépôt et à la diffusion de documents scientifiques de niveau recherche, publiés ou non, émanant des établissements d'enseignement et de recherche français ou étrangers, des laboratoires publics ou privés. 


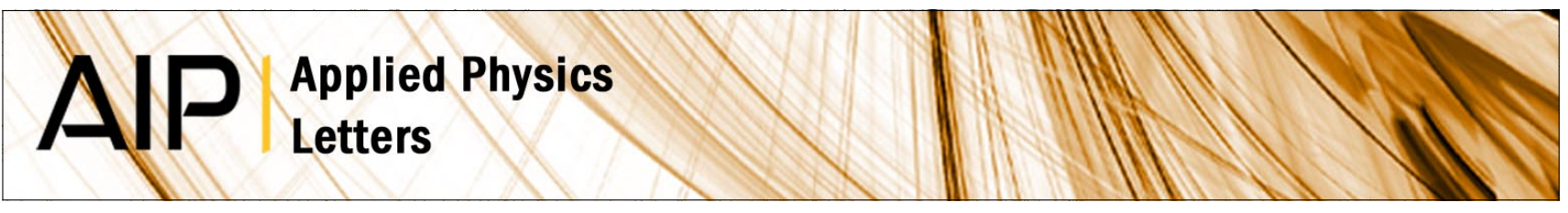

\section{Sharp acoustic multipolar-resonances in highly monodisperse emulsions}

Thomas Brunet, Simon Raffy, Benoit Mascaro, Jacques Leng, Régis Wunenburger et al.

Citation: Appl. Phys. Lett. 101, 011913 (2012); doi: 10.1063/1.4733615

View online: http://dx.doi.org/10.1063/1.4733615

View Table of Contents: http://apl.aip.org/resource/1/APPLAB/v101/i1

Published by the American Institute of Physics.

\section{Related Articles}

Size dependent $\mathrm{Cu}$ dielectric function for plasmon spectroscopy: Characterization of colloidal suspension generated by fs laser ablation

J. Appl. Phys. 112, 054319 (2012)

Particle size and density of a slurry from ultrasonic backscattering measurements at a solid interface

Rev. Sci. Instrum. 83, 095101 (2012)

Tuning effective interactions close to the critical point in colloidal suspensions

J. Chem. Phys. 137, 084903 (2012)

A method to measure specific absorption rate of nanoparticles in colloidal suspension using different configurations of radio-frequency fields

Appl. Phys. Lett. 101, 083118 (2012)

Krylov subspace methods for computing hydrodynamic interactions in Brownian dynamics simulations J. Chem. Phys. 137, 064106 (2012)

\section{Additional information on Appl. Phys. Lett.}

Journal Homepage: http://apl.aip.org/

Journal Information: http://apl.aip.org/about/about_the_journal

Top downloads: http://apl.aip.org/features/most_downloaded

Information for Authors: http://apl.aip.org/authors

\section{ADVERTISEMENT}

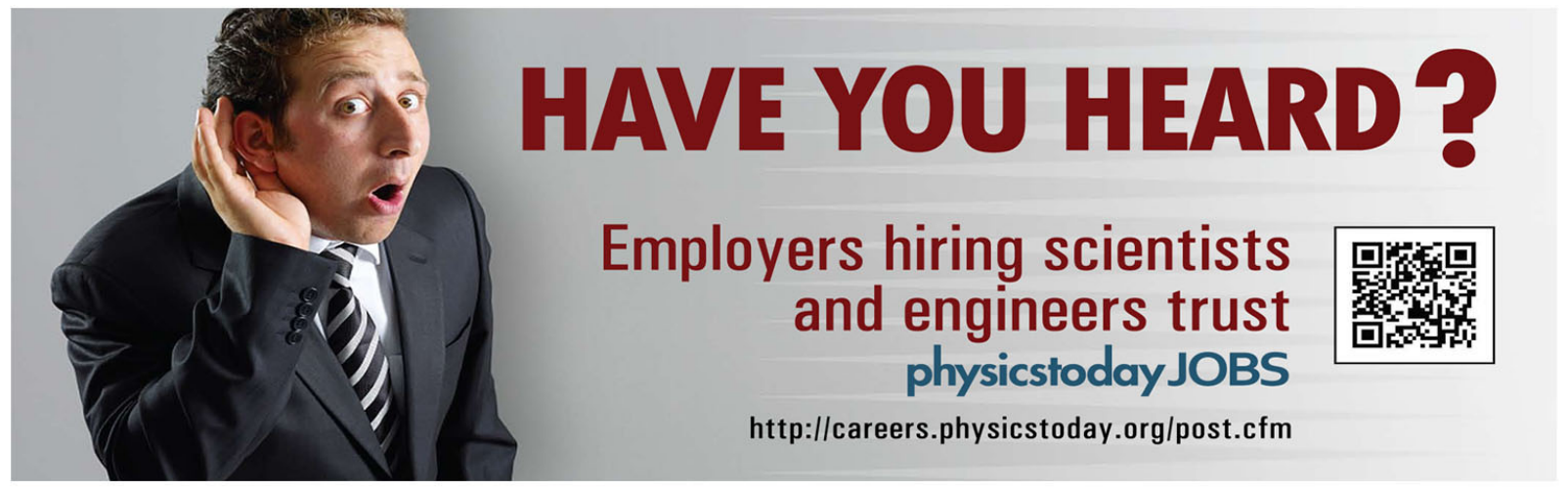




\title{
Sharp acoustic multipolar-resonances in highly monodisperse emulsions
}

\author{
Thomas Brunet, ${ }^{1,2, a)}$ Simon Raffy, ${ }^{1,3}$ Benoit Mascaro, ${ }^{1,2,3}$ Jacques Leng, ${ }^{1,4}$ \\ Régis Wunenburger, ${ }^{1,5}$ Olivier Mondain-Monval, ${ }^{1,3}$ Olivier Poncelet, ${ }^{1,2}$ \\ and Christophe Aristégui ${ }^{1,2}$ \\ ${ }^{1}$ CNRS, F-33400 Talence, France \\ ${ }^{2}$ Univ. Bordeaux, I2M, UMR 5295, F-33405 Talence, France \\ ${ }^{3}$ Univ. Bordeaux, CRPP, UPR 8641, F-33600 Pessac, France \\ ${ }^{4}$ Univ. Bordeaux, LOF, UMR 5258, F-33608 Pessac, France \\ ${ }^{5}$ Univ. Bordeaux, LOMA, UMR 5798, F-33405 Talence, France
}

(Received 22 May 2012; accepted 18 June 2012; published online 6 July 2012)

\begin{abstract}
We report the achievement of highly monodisperse emulsions exhibiting about ten acoustic Mie resonances. Thanks to robotics, the effective acoustic properties of such strongly scattering media can be precisely targeted by means of the production of calibrated (random) liquid-droplets. Ultrasonic experiments are compared, with an excellent quantitative agreement, to theoretical predictions derived within the framework of the independent scattering approximation. The dependence of the sound speed and of the acoustic attenuation on both the size and the volume fraction of droplets is quantitatively examined for dilute and more concentrated emulsions, and is presented in a dimensionless way. (C) 2012 American Institute of Physics. [http://dx.doi.org/10.1063/1.4733615]
\end{abstract}

Wave propagation and scattering in random media have been intensely studied for two decades. ${ }^{1}$ In this context, scattering resonances play an important role by affecting the macroscopic (effective) properties of such complex media. In acoustics, the different modal resonances of particles are driven by the shape and the physical properties of the scatterers. The experimental study of such resonances in the Mie scattering regime, i.e., when the wavelength is comparable with the size of the scatterers $(k a \sim 1)$, is a hard task due to the difficulties associated with observing them at the high frequencies required. ${ }^{2}$ Finding a model system exhibiting a wide collection of observable multipolar resonances is challenging because many drastic conditions are required to their emergence in the coherent-wave dispersive properties. One of them is the monodispersity in both size and shape of the random medium, as obtained with suspensions of polystyrene microspheres. ${ }^{3}$ Indeed, polydispersity effects tend to smooth the spectral resonant features. ${ }^{4}$ In case of huge mechanical contrasts between the scatterers and the surrounding medium, such latter restriction becomes less important as for bubbly media. However, the high compressibility of the air bubbles induces such a strong monopolar resonance, so-called the Minnaert resonance, which the others modal resonances are not observable. ${ }^{5,6}$ As another example, inertial effects of heavy core-shell particles ${ }^{7}$ or membranetype inclusions ${ }^{8}$ favor the dipolar resonance to the detriment of the others modes.

In place of solid particles or gas bubbles, liquid droplets randomly dispersed in a fluid matrix could provide an appropriate system to reveal multipolar scattering resonances, provided the structure of such random media is perfectly controlled (droplet size, polydispersity, etc). Practically, ultrasound scattering by emulsions has been mostly considered in the long-wavelength regime. ${ }^{9}$ Although resonant scattering from spherical cavities in viscoelastic media has

a)Electronic mail: thomas.brunet@u-bordeaux1.fr. already been theoretically considered, ${ }^{10}$ no experimental results have been reported on the resonant regime occurring in emulsions.

In this letter, we report the realization of highly monodisperse emulsions that exhibit sharp multipolar resonances. The macroscopic (effective) acoustic properties of such random media can be frequency-driven by tuning the Mie resonances of the droplets (essentially by fixing the droplet radius). As controlled properties, we mainly think here about the frequency-location of the attenuation peaks along with their magnitude, which depends on the volume fraction of droplets. We also aim at prospecting the sensitivity of the sound speed to the resonant features that can be experimentally assessed.

To examine precisely the multi-resonant behavior of emulsions, a low absorption suspending fluid is required. Thus, we used an aqueous polymer gel (Carbopol ${ }^{\circledR}$ ETD $2050,0.2 \mathrm{wt} \%),{ }^{11}$ of which the acoustic properties are very close to those of water in the megahertz range we investigated. The sound speed $v_{0}$ and the acoustic attenuation $\alpha_{0}$ in this water-based gel were measured (see below) and fitted as $1492 \mathrm{~m} \mathrm{~s}^{-1}$ and $8.10^{-5} \mathrm{MHz}^{-2} \mathrm{~mm}^{-1}$, respectively, while its mass density $\rho_{0}$ is $1.005 \mathrm{~g} \mathrm{~cm}^{-3}$.

In order to enhance Mie scattering resonances, high mechanical contrasts between the fluid matrix and the liquid inclusions are required. Thus, we used fluorinated-oil droplets (FC-40, Fluorinert ${ }^{\circledR}$ ) possessing a rather low sound speed $v_{1}$ for a pure liquid (about $640 \mathrm{~m} \mathrm{~s}^{-1}$ ) and a high mass density $\left(\rho_{1}=1.85 \mathrm{~g} \mathrm{~cm}^{-3}\right)$. Note that the yield stress $(\sim 7 \mathrm{~Pa})$ of the aqueous gel-matrix circumvents the sedimentation of the heavy oil-droplets.

As previously done for bubbly media, ${ }^{12}$ periodic rows of regularly spaced fluorinated-oil droplets were achieved thanks to robotics. The fluorinated oil was continuously injected within the aqueous gel-matrix by means of a moving syringe. Typically, $100 \mu \mathrm{m}$-radius droplets were obtained with fast displacements $\left(\sim 100 \mathrm{~mm} \mathrm{~s}^{-1}\right)$ and low rates of oil 

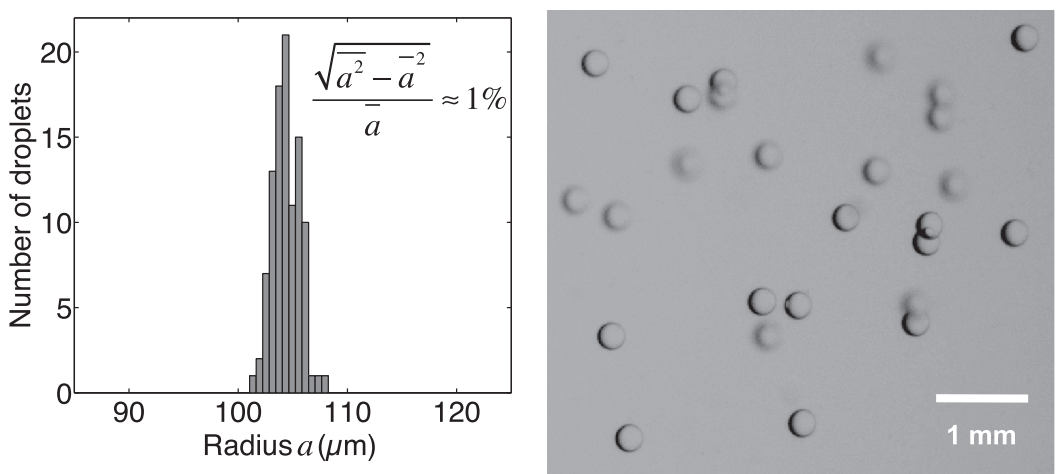

FIG. 1. Histogram of the droplet radius distribution for sample 1 obtained by optical measurements on 132 droplets. The average radius droplet $\bar{a}$ is $104 \mu \mathrm{m}$ and the sizepolydispersity is about $1 \%$. The corresponding volume fraction $\Phi_{v}$ of droplets displayed in the image is about $0.25 \%$.

flow $\left(\sim 10 \mu \mathrm{lmin}^{-1}\right)$. This immiscible-in-water oil is much more stable than a gas, which makes the emulsion production faster and its conservation longer than those of a bubbly medium. In addition, we obtained an excellent monodispersity for both size and spherical-shape of droplets as illustrated in Fig. 1. The droplet-size distribution was optically characterized by the analysis of about a hundred droplets. The radius measurements were made with a one pixel accuracy, i.e., $1 \mu \mathrm{m}$. The size-polydispersity, of about $1 \%$, was determined from the discrete distribution of the droplet radius as shown in Fig. 1. Two samples were prepared: a dilute emulsion (sample 1 with $\Phi_{v}=0.25 \%$ and $\bar{a}=104 \mu \mathrm{m}$ ) and a more concentrated one (sample 2 with $\Phi_{v}=6 \%$ and $\bar{a}=175 \mu \mathrm{m})$. The volume fractions of the fluorinated oildroplets were estimated from the liquid quantity injected

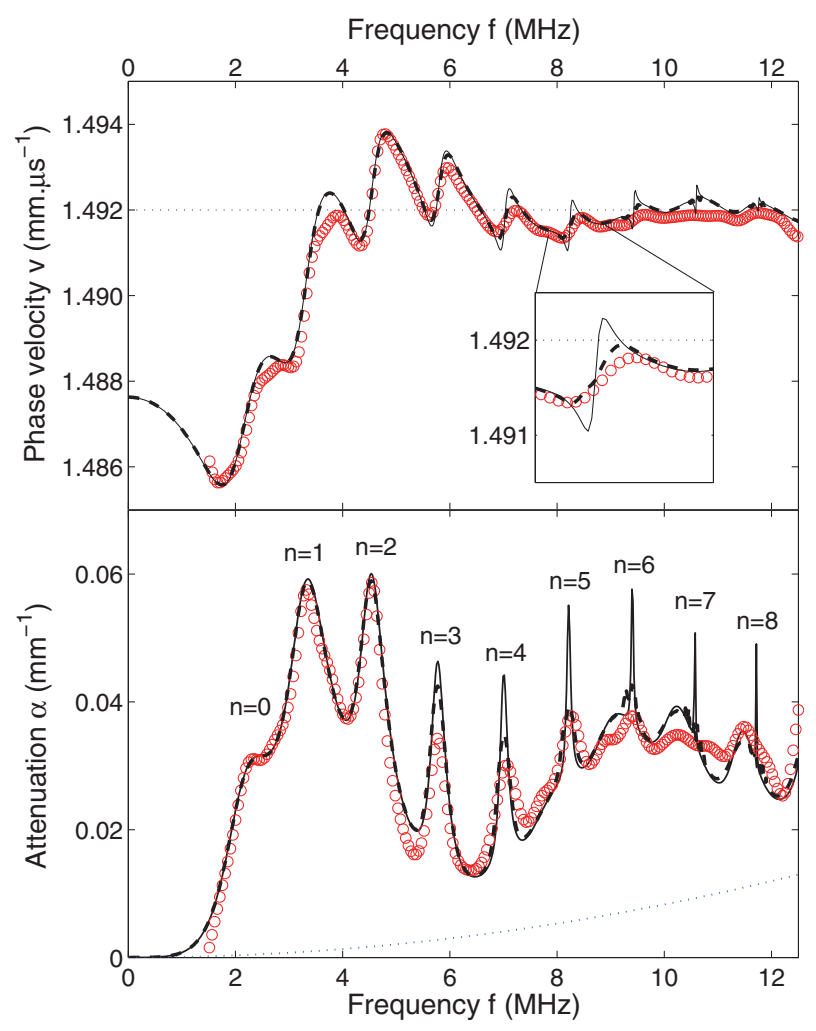

FIG. 2. Phase velocity (top) and attenuation (bottom) measured in sample 1 (red open circles). Theoretical predictions from the ISA for a monodisperse emulsion (solid line) and for a $1 \%$-polydisperse emulsion (dashed line, see the discrete radius-distribution in Fig. 1) with $\Phi_{v}=0.23 \%$ and $\bar{a}=104 \mu \mathrm{m}$. Fitted measurements of acoustic properties of the pure aqueous gel-matrix (dotted line). within the aqueous gel-matrix. Finally, note that the disorder will be due to the transfer of the (regularly spaced) emulsion into the cell devoted to acoustic measurements, with no change in droplet size.

For dilute emulsions such as sample 1, ultrasonic measurements were carried out in a large water tank $\left(80 \times 40 \times 30 \mathrm{~cm}^{3}\right)$ by multi-echo spectroscopy. ${ }^{13}$ An ultrasound pulse was generated by a broadband pulser/receiver connected to a half-inch $10-\mathrm{MHz}$ longitudinal-wave transducer with a broad frequency-bandwidth (1-12 MHz). The emulsion was poured into a polystyrene cell $\left(10 \times 10 \mathrm{~cm}^{2}\right)$ and placed at the Rayleigh distance of the transducer. The cell was thick enough $(15 \mathrm{~mm})$ to separate the multiple echoes reflected by the walls. By means of this reflexion technique, we first measured the complex-valued wavenumber $k_{0}=k_{0}^{\prime}+\mathrm{i} k_{0}^{\prime \prime}$ of the pure polymer gel-matrix $\left(k_{0}^{\prime}=\frac{\omega}{v_{0}}\right.$ and $k_{0}^{\prime \prime}=\alpha_{0}$ with $\omega=2 \pi f$ standing for the angular frequency) as shown in Fig. 2 (dotted line). Then, we measured both the phase velocity $v$ and the attenuation coefficient $\alpha$ of the coherent wave propagating in emulsions. In such scattering media, the incoherent part of the ultrasonic field could be non negligible. ${ }^{14}$ However, as we used large transducers, of which the diameters are about 10 times larger than the longest wavelength $\lambda_{0}(\sim 1.5 \mathrm{~mm}$ at $1 \mathrm{MHz})$ involved in our experiments, a "self-averaging" on their surfaces provides an access to a good estimate of the coherent part from only one configuration of the disorder.

The ultrasonic measurements reported in Fig. 2 evidence several sharp attenuation peaks associated with fast phasevelocity variations. Since the volume fraction of droplets is quite low $\left(\Phi_{v} \leq 1 \%\right)$, we analyzed the experimental results within the framework of the independent scattering approximation (ISA). ${ }^{1}$ This approach is equivalent to the Foldy's model and does not take into account the coupling between scatterers. ${ }^{15}$ Thus, the effective wavenumber $k_{\text {eff }}$ of the coherent pressure wave propagating in such random mixture is given by:

$$
k_{e f f}^{2}=\left(\frac{\omega}{v}+\mathrm{i} \alpha\right)^{2}=k_{0}^{2}+4 \pi \eta f_{s}(0)
$$

where $\eta$ is the number of droplets per unit volume $\left(\Phi_{v}=\frac{4}{3} \pi a^{3} \eta\right)$ and $f_{s}(0)=\frac{1}{\mathrm{i} k_{0}} \sum_{n=0}^{\infty}\{2 n+1\} S_{n}$ the forward scattering function. ${ }^{16}$ The complex-valued coefficients $S_{n}$, which depend on both $k_{0} a$ and $k_{1} a$, and on the ratios $\frac{\rho_{1}}{\rho_{0}}$ 
and $\frac{v_{1}}{v_{0}}$, are the scattering modal coefficients of a single droplet embedded in the gel-matrix. The index $n$ refers to the $n$th mode, e.g., $n=0, n=1$, and $n=2$ are associated with the monopolar, dipolar, and quadrupolar vibration modes, respectively. The resonance frequencies of each mode fix the locations of the attenuation peaks as well as the corresponding maxima in the phase-velocity variations. For example, we observe in Fig. 2 at 2.3, 3.3, and 4.5 MHz the signature of the very first resonances of the monopolar, dipolar, and quadrupolar modes.

For comparison, both the phase velocity and the attenuation coefficient predicted from Eq. (1) have been superimposed on the experimental measurements performed on sample 1 (Fig. 2). By slightly tuning the sound speed $v_{1}$ of the fluorinated oil, we made the predicted absorption peak locations match precisely the experimental ones for all resonant peaks. The best agreement is found for $v_{1}=645 \mathrm{~m} \mathrm{~s}^{-1}$. Subsequently, the attenuation level is recovered by adjusting the value of the droplet volume-fraction $\left(\Phi_{v}=0.23 \%\right)$. Note that possible misalignment in the acoustical set-up may induce a deviation on the measured attenuation level at high frequencies. However, the resonance peak locations should not be affected because they mainly depend on the droplet size.

As a result, the ISA well-fits the measured attenuation peaks as well as the phase-velocity dispersion in the Mie scattering regime, especially for the first modes $(n \leq 2)$. Obviously the narrowest absorption peaks, which are theoretically expected at higher frequencies, are not experimentally recovered (Fig. 2) because the actual emulsion is not strictly monodisperse. In spite of a very low sizepolydispersity $(\sim 1 \%)$, taking into account the discrete radius distribution shown in Fig. 1 in the ISA $\left(k_{\text {eff }}^{2}=k_{0}^{2}\right.$ $\left.+\sum_{a_{i}} 4 \pi \eta\left(a_{i}\right) f_{s}\left(0, a_{i}\right)\right)$ leads to a much better description of the experimental data beyond $8 \mathrm{MHz}$. Note that for these higher frequencies, the phase-velocity variations become weaker and weaker (less than $1 \mathrm{~m} / \mathrm{s}$, i.e., less than $0.1 \%$ ) but still remain observable and in good agreement with theoretical predictions as shown in the zoom of Fig. 2.

We also investigated the acoustic response of a more concentrated emulsion with a different mean-radius: sample $2\left(\Phi_{v}=6 \%\right.$ and $\left.\bar{a}=175 \mu \mathrm{m}\right)$. As the volume fraction of droplets is appreciably increased in comparison with sample 1 , the scattering effects induce much stronger losses and so higher attenuation. Therefore, in order to still make accurate acoustic measurements, there is a need to reduce the distance of propagation. Thus, we used a through-transmission configuration with a pair of 5-MHz longitudinal-wave transducers, and a thinner measurement cell $(5 \mathrm{~mm})$. The same resonant features are observable for sample 2 (Fig. 3), as those reported for sample 1 (Fig. 2). Note that the frequencyshift of the attenuation peaks to lower frequencies is due to the larger droplet-radius of sample 2. More importantly, the higher volume fraction of droplets induces stronger effects in sample 2 such as large attenuation peaks $\left(\sim 1 \mathrm{~mm}^{-1}\right)$ and large phase-velocity dispersion $(\sim 13 \%)$ as illustrated by the temporal signal shown in the inset of Fig. 3. The acoustic measurements also show at around $3 \mathrm{MHz}$ an increase of the

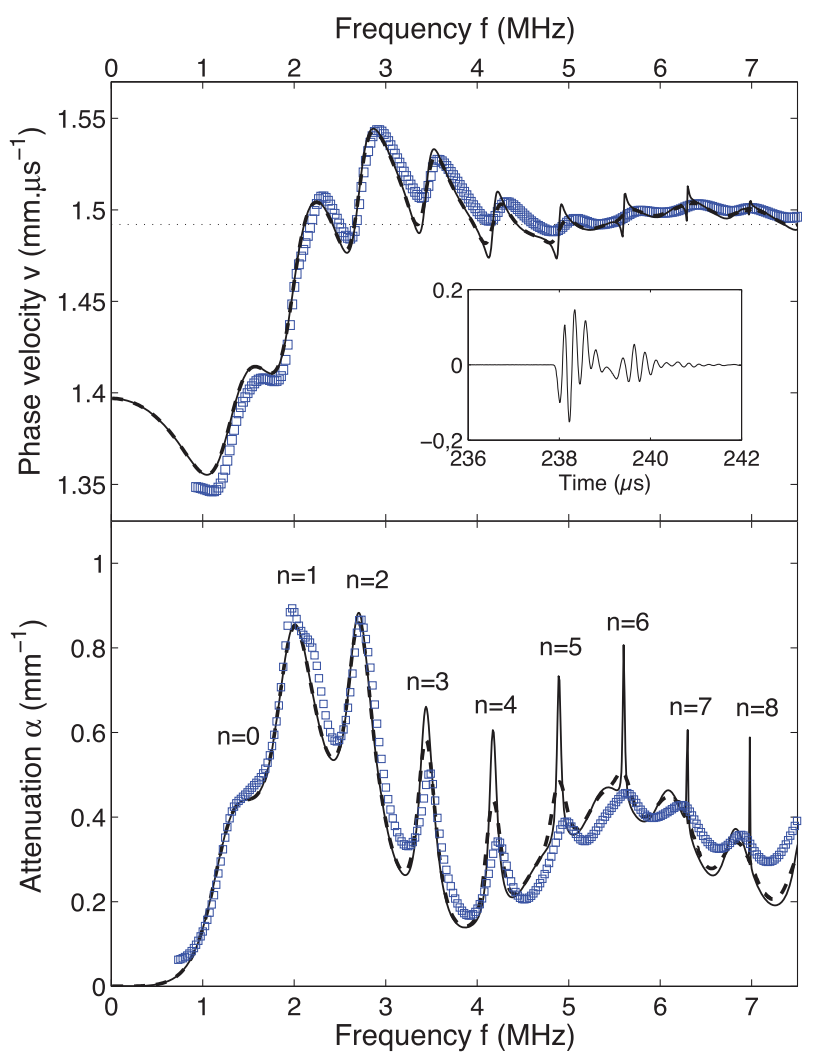

FIG. 3. Phase velocity (top) and attenuation (bottom) measured in sample 2 (blue open squares). Theoretical predictions from the ISA for a monodisperse emulsion (solid line) and for a 1\%-polydisperse emulsion (dashed line) with $\Phi_{v}=5.8 \%$ and $\bar{a}=175 \mu \mathrm{m}$. Inset: temporal signal transmitted through sample 2 without space-averaging.

sound speed of about $50 \mathrm{~m} \mathrm{~s}^{-1}$ compared to the pure gelmatrix. As done for sample 1, the best agreement between the experimental results and the theoretical predictions has been found by adjusting the value of the droplet volumefraction of sample $2\left(\Phi_{v}=5.8 \%\right)$.

At last, we focus our attention on the variations of the emulsion acoustic properties induced by the scattering effects only. Thus, we use dimensionless expression for the phase-velocity variation $\left(\Delta v=v-v_{0}\right)$ normalized by the phase velocity $v_{0}$ of the pure aqueous gel-matrix and reduced to the volume fraction $\Phi_{v}$ of droplets. In the same way, we consider the attenuation increase $\left(\Delta \alpha=\alpha-\alpha_{0}\right)$ per wavelength $\lambda_{0}\left(=2 \pi / k_{0}^{\prime}\right)$ and also reduced to the volume fraction $\Phi_{v}$ of droplets. On one hand, Eq. (1) can be linearized with the assumption $\alpha \ll \frac{\omega}{v}$, provided the emulsions are not too much concentrated. On the other hand, the absorption within the pure gel-matrix is low enough to also consider that $\alpha_{0} \ll \frac{\omega}{v_{0}}$. Thus, the dimensionless acoustic parameters can be estimated from the ISA model as following:

$$
\begin{gathered}
\frac{1}{\Phi_{v}}\left(\frac{\Delta v}{v_{0}}\right)=\frac{-3}{2\left(k_{0}^{\prime} a\right)^{3}} \operatorname{Im}\left[\sum_{n=0}^{\infty}\{2 n+1\} S_{n}\left(k_{0}^{\prime} a\right)\right] \\
\frac{1}{\Phi_{v}}\left(\frac{\Delta \alpha}{k_{0}^{\prime}}\right)=\frac{-3}{2\left(k_{0}^{\prime} a\right)^{3}} \operatorname{Re}\left[\sum_{n=0}^{\infty}\{2 n+1\} S_{n}\left(k_{0}^{\prime} a\right)\right] .
\end{gathered}
$$

Due to the form of the scattering coefficients $S_{n}$, the dimensionless phase-velocity variations (Eq. (2a)) and the 


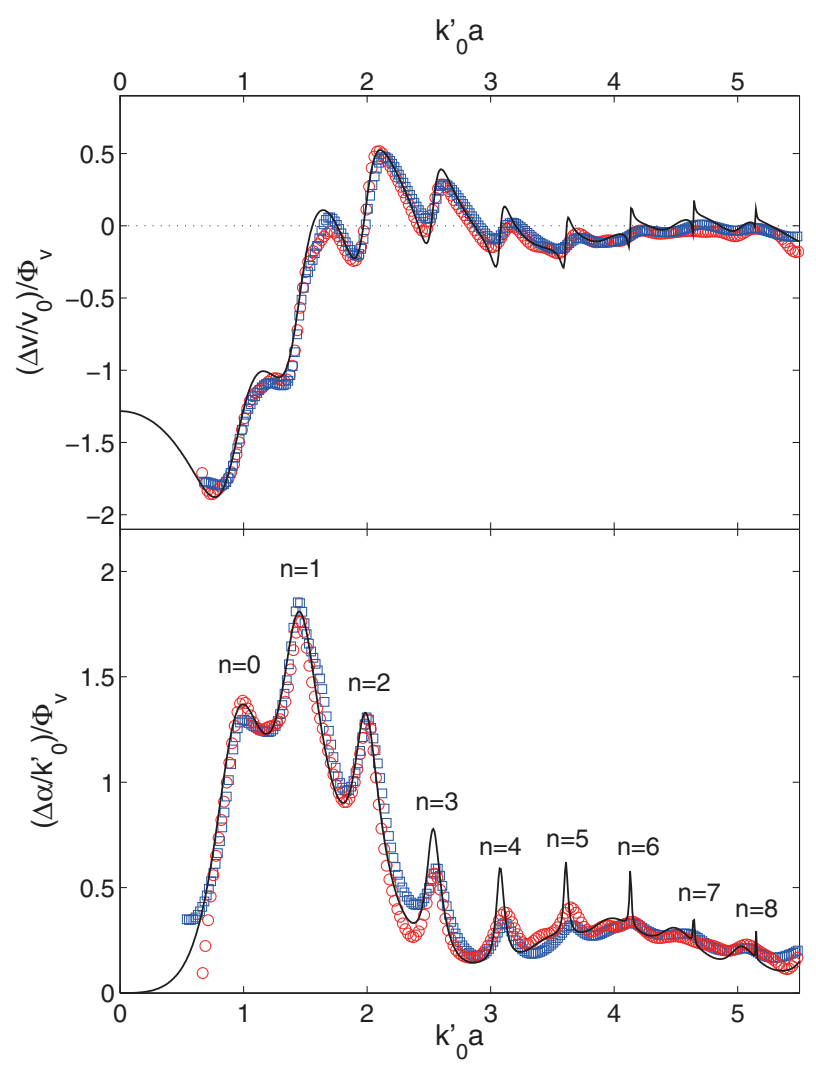

FIG. 4. Dimensionless phase velocity (top) and dimensionless attenuation (bottom) versus reduced frequency measured in sample 1 (red open circles) and sample 2 (blue open squares). Theoretical predictions from Eq. (2a) and (2b) for a monodisperse emulsion made of fluorinated oil-droplets randomly dispersed within an aqueous gel-matrix (solid line).

dimensionless attenuation increase (Eq. (2b)) are independent of both the volume fraction $\Phi_{v}$ and the droplet radius $a$. Thus, whatever the values of the latter parameters, for reasonably small $\Phi_{v}$, the experimental data should follow a unique master theoretical curve for the given couple of materials here studied, i.e., fluorinated oil-droplets randomly dispersed within an aqueous gel-matrix. Fig. 4 shows that the two experimental curves (open symbols) are wellsuperimposed on the master theoretical curve (solid line). The phase-velocity dispersion, the attenuation level, and the resonance-frequency locations are well-recovered for both samples. The first resonances are clearly evidenced whatever the volume fraction and the size of the droplets. At high frequencies, the size-polydispersity of both the samples $(\sim 1 \%)$ is responsible for the spreading of the resonance peaks, which are no longer observable beyond $k_{0}^{\prime} a \geq 5$.

In summary, we have presented experimental evidence about acoustic multipolar resonances occurring in precisely controlled monodisperse emulsions with high sound-speed contrasts in the Mie scattering regime. The independent scattering approximation well-describes the acoustic resonant response of these emulsions for $1 \leq k_{0}^{\prime} a \leq 5$, even for moderate volume fractions of scatterers of about few percents. These strongly scattering media might be used as functional materials with frequency-controlled acoustic properties (sound speed and acoustic attenuation). Slow/fast wave effects and ultra-damping could be achieved if the volume fraction of oil-droplets is appreciably increased. However, the tools for the design of highly concentrated emulsions need then to consider interactions between oil-droplets through the modeling of multiple scattering. At last, these results may have an interest in the metamaterial community. In fact, as mentioned by Li et al. (see Chap. 8 in Ref. 17), one possibility to obtain metamaterial features such as "negative mass density" and/or "negative compressibility" is to create strong Mie-type resonances. That should be achieved by finding two components with huge sound-speed contrasts, i.e., much higher than those presented in this paper.

We thank A. Aradian for initial stimulating discussions, and Le Conseil Régional d'Aquitaine for PhD grant and fundings through the cluster Advanced Materials in Aquitaine (GIS-AMA). This work is supported by Grant USAF FA8655-11-M-4006 and by Grant ANR-2011-BS0902101 Metakoustik-Aerospace Valley. We are also grateful to the reviewer's comments and advices.

${ }^{1} \mathrm{P}$. Sheng, Introduction to Wave Scattering, Localization, and Mesoscopic Phenomena (Springer, Berlin, 2006).

${ }^{2}$ R. E. Challis, M. J. W. Povey, M. L. Mather, and A. K. Holmes, Rep. Prog. Phys. 68, 1541 (2005).

${ }^{3}$ C. S. Hall, J. N. Marsh, M. S. Hughes, J. Mobley, K. D. Wallace, J. G. Miller, and G. H. Brandenburger, J. Acoust. Soc. Am. 101, 1162 (1997).

${ }^{4}$ I. Alig and D. Lellinger, J. Appl. Phys. 72, 5565 (1992).

${ }^{5}$ V. Leroy, A. Bretagne, M. Fink, H. Willaime, P. Tabeling, and A. Tourin, Appl. Phys. Lett. 95, 171904 (2009).

${ }^{6}$ A. Bretagne, A. Tourin, and V. Leroy, Appl. Phys. Lett. 99, 221906 (2011).

${ }^{7}$ Z. Liu, X. Zhang, Y. Mao, Y. Y. Zhu, Z. Yang, C. T. Chan, and P. Sheng, Science 289, 1734 (2000).

${ }^{8}$ Z. Yang, H. M. Dai, N. H. Chan, G. C. Ma, and P. Sheng, Appl. Phys. Lett. 96, 041906 (2010).

${ }^{9}$ D. J. McClements and M. J. W. Povey, J. Phys. D: Appl. Phys. 22, 38 (1989).

${ }^{10}$ G. C. Gaunaurd and H. Uberall, J. Acoust. Soc. Am. 63, 1699 (1978).

${ }^{11}$ A. M. V. Putz and T. I. Burghelea, Rheol. Acta 48, 673 (2009).

${ }^{12}$ V. Leroy, A. Strybulevych, J. H. Page, and M. G. Scanlon, J. Acoust. Soc. Am. 123, 1931 (2008).

${ }^{13}$ T. Norisuye, S. Sasa, K. Takeda, M. Kohyama, and Q. Tran-Cong-Miyata, Ultrasonics 51, 215 (2011).

${ }^{14}$ A. Derode, A. Tourin, and M. Fink, Phys. Rev. E 64, 036605 (2001).

${ }^{15}$ L. L. Foldy, Phys. Rev. 67, 107 (1945).

${ }^{16}$ C. Aristegui and Y. C. Angel, Wave Motion 44, 153 (2007).

${ }^{17}$ Physics of Negative Refraction and Negative Index Materials, edited by C. M. Krowne and Y. Zong (Springer, Berlin, 2007). 